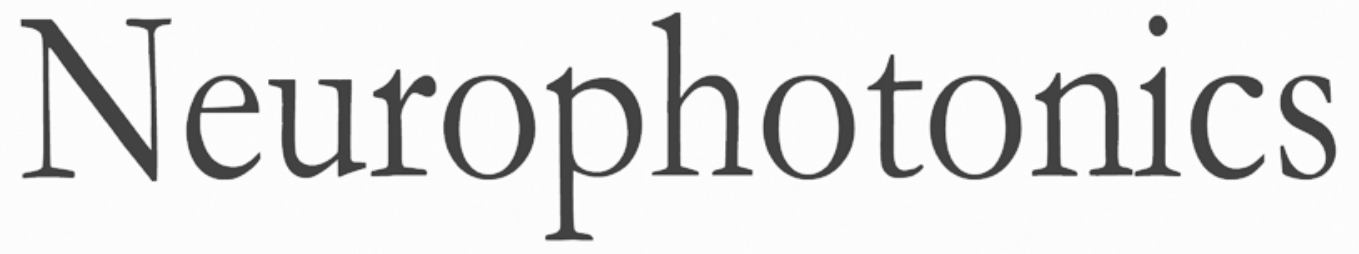

\title{
Comprehensive optical and data management infrastructure for high- throughput light-sheet microscopy of whole mouse brains
}

M. Caroline Müllenbroich

Ludovico Silvestri

Leonardo Onofri

Irene Costantini

Marcel van't Hoff

Leonardo Sacconi

Giulio Iannello

Francesco S. Pavone

\section{SPIE.}




\title{
Comprehensive optical and data management infrastructure for high-throughput light-sheet microscopy of whole mouse brains
}

\author{
M. Caroline Müllenbroich, ${ }^{\text {a,b }}$ Ludovico Silvestri, ${ }^{a, c}$ Leonardo Onofri, ${ }^{a, d}$ Irene Costantini, ${ }^{a}$ Marcel van’t Hoff, ${ }^{a, b}$ \\ Leonardo Sacconi, ${ }^{a, c}$ Giulio lannello, ${ }^{d}$ and Francesco S. Pavone ${ }^{a, b, c, e, *}$ \\ aUniversity of Florence, European Laboratory for Non-Linear Spectroscopy, Via Nello Carrara 1, Sesto Fiorentino 50019, Italy \\ bUniversity of Florence, Department of Physics and Astronomy, Via Sansone 1, Sesto Fiorentino 50019, Italy \\ 'National Institute of Optics, National Research Council, Via Nello Carrara 1, Sesto Fiorentino 50019, Italy \\ dUniversity Campus Bio-Medico of Rome, v. Alvaro del Portillo 21, Roma 00128, Italy \\ eInternational Centre for Computational Neurophotonics, Via Nello Carrara 1, Sesto Fiorentino 50019, Italy
}

\begin{abstract}
Comprehensive mapping and quantification of neuronal projections in the central nervous system requires high-throughput imaging of large volumes with microscopic resolution. To this end, we have developed a confocal light-sheet microscope that has been optimized for three-dimensional (3-D) imaging of structurally intact clarified whole-mount mouse brains. We describe the optical and electromechanical arrangement of the microscope and give details on the organization of the microscope management software. The software orchestrates all components of the microscope, coordinates critical timing and synchronization, and has been written in a versatile and modular structure using the LabVIEW language. It can easily be adapted and integrated to other microscope systems and has been made freely available to the light-sheet community. The tremendous amount of data routinely generated by light-sheet microscopy further requires novel strategies for data handling and storage. To complete the full imaging pipeline of our high-throughput microscope, we further elaborate on big data management from streaming of raw images up to stitching of 3-D datasets. The mesoscale neuroanatomy imaged at micron-scale resolution in those datasets allows characterization and quantification of neuronal projections in unsectioned mouse brains. ๑ The Authors. Published by SPIE under a Creative Commons Attribution 3.0 Unported License. Distribution or reproduction of this work in whole or in part requires full attribution of the original publication, including its DOI. [DOI: 10.1117/1.NPh.2.4 .041404]
\end{abstract}

Keywords: light-sheet microscopy; selective plane illumination microscopy; whole brain imaging; data management; software management; high-throughput imaging.

Paper 15012SSR received Feb. 20, 2015; accepted for publication May 14, 2015; published online Jun. 29, 2015.

\section{Introduction}

The highly ambitious project of mapping and understanding each and every neuronal projection in the whole brain has been moved to feasible reality by the recent advent of lightsheet fluorescent microscopy (LSFM). ${ }^{1-3}$ With this technique, three-dimensional (3-D) datasets can be acquired with a resolution that is high enough to identify neurons and their dendritic and axonal features in time scales which are no longer the bottleneck of high-throughput acquisition. In LSFM, the sample is illuminated with a thin sheet of light confined into the focal plane of the detection objective, which collects the fluorescence emission along an axis perpendicular to the illumination plane. ${ }^{4}$ This technique drastically reduces the imaging acquisition time by recording millions of pixels in parallel due to its wide-field detection and affords intrinsic optical sectioning due to the lightsheet illumination. Consequently, fluorophores outside the light sheet are neither bleached nor contribute blurring out-of-focus noise. Together, these attributes cause a high spatiotemporal resolution and an excellent signal-to-noise ratio (SNR) in LSFM.

*Address all correspondence to: Francesco S. Pavone, E-mail: francesco .pavone@unifi.it
Several challenges remain to be overcome, however, to allow fast and, most of all, systematic production of reliable datasets and their meaningful interpretation to further our understanding of neuronal networks. Those challenges include fast, cheap, and reproducible sample preparation and mounting, automated image acquisition and, most crucially, storage, interpretation, and analysis of the vast datasets light-sheet microscopy routinely produces." ${ }^{5,6}$ Mapping and understanding of this "big data" is an immense task that requires the expertize of computer scientists to employ fully automated, scalable postprocessing, for example, to carry out blood vessel segmentation or cell counting. ${ }^{7}$ On the other hand, the imaging of large, intrinsically opaque samples in LSFM necessitates clearing protocols based on refractive index matching, which render the tissue transparent. ${ }^{8,9}$ Therefore, technological advances in LSFM need to be matched by novel development in the area of information and biotechnology.

Here, we will present a state-of-the-art light-sheet microscope, as it is implemented in our lab, that is especially wellsuited to acquire 3-D datasets of clarified and structurally intact mouse brains. The LSFM features double-sided illumination with a digitally scanned light sheet and a sample chamber, which has been specifically designed for the the imaging of large $\left(>1 \mathrm{~cm}^{2}\right)$ immersed and freely movable samples. After 
briefly introducing the optical setup, we explain how to prepare and mount the samples for stable 3-D imaging for several days. In addition, a full description of the control hardware and software is presented. The microscope software coordinates every microscope component and ensures critical timing and synchronization between those components. We further give a systematic and robust approach to handle and store light-sheet microscopy data of several terabytes. To conclude the comprehensive and high-throughput imaging pipeline, we furthermore elaborate on stitching of large 3-D datasets. The imaging capabilities of our light-sheet microscope are exemplified with tomographies of whole transgenic mouse brains.

\section{Optical Setup}

\subsection{Laser Unit}

The custom-made confocal light-sheet microscope is equipped with five linearly polarized, continuous-wave lasers for fluorescence excitation [Fig. 1(a)]. The wavelengths were chosen to excite the most common fluorophores (see Table 1 for the manufacturer and specifications of microscope components). The beam from each laser is first collimated and expanded with a telescope made of singlet lenses ( $f 140$ and $f 200)$ and then combined into a common path with a mirror and a long-pass filter (Semrock, LaserMUX ${ }^{\mathrm{TM}}$ series). From here on, all lenses are achromatic doublets coated for the visible spectrum. An acousto-optical tunable filter (AOTF) acts as fast ( $\mu \mathrm{s})$ electronically tunable filter and intensity modulator. Due its nonlinear response, we measured the AOTF light transmittance for each wavelength as a function of radio-frequency amplitude and determined a lookup table to linearize the output. The zeroorder light is blocked by an iris. A 1:1 telescope ( $f 150$ and $f 150$ ) is used to recollimate the beam after the AOTF. An electro-optical laser modulator (EOM) acts like a waveplate with electronically controlled retardation and rapidly rotates (few hundreds of nanoseconds) the input polarization of the excitation light by $90 \mathrm{deg}$. A digital line is used to switch between the voltage levels, allowing control of the frequency with which the polarization is changed (see also Sec. 3.2 on timing).
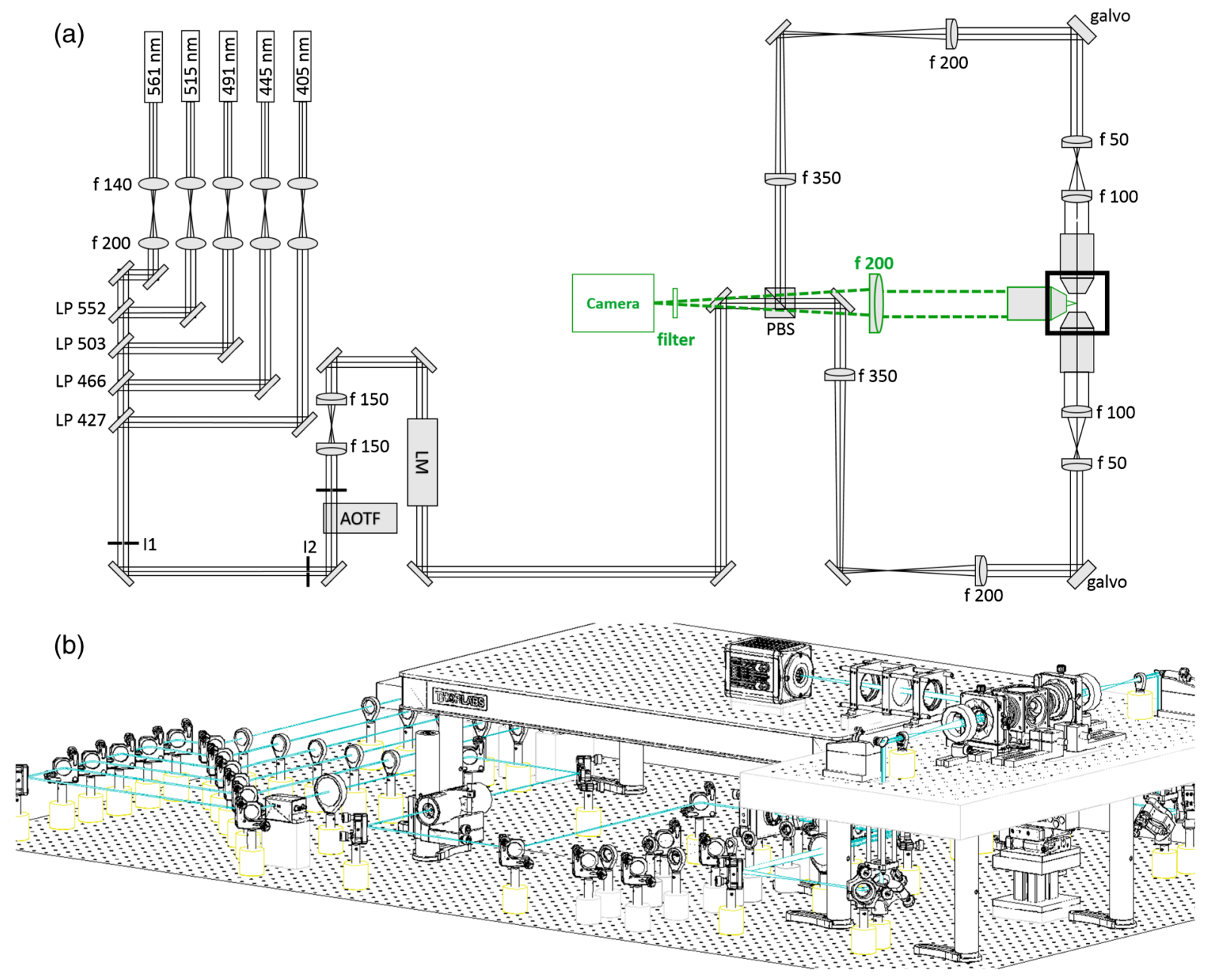

Fig. 1 (a) Schematic of the light path. The galvo scanners are mounted above periscopes. LP: long-pass filter, I: iris, AOTF: acousto-optical tunable filter, LM: laser modulator, PBS: polarization beam splitter. Green dotted: detection path. (b) Oblique view of the microscope. A custom-made breadboard serves to mount the sample chamber and objectives at an elevated height and features two circular holes at the edges for the periscopes and a large central cut-out for the translation stages. A second breadboard is used for the camera. 
Table 1 Overview of microscope components.

\begin{tabular}{|c|c|c|c|}
\hline Component & Manufacturer & Part\# & Specifications \\
\hline \multirow[t]{5}{*}{ Lasers } & Cobolt AB, Sweden & MLD & $405 \mathrm{~nm}, 80 \mathrm{~mW}$, s-polarized \\
\hline & & MLD & $445 \mathrm{~nm}, 50 \mathrm{~mW}$, s-polarized \\
\hline & & Calypso & $491 \mathrm{~nm}, 50 \mathrm{~mW}$, s-polarized \\
\hline & & Fandango & $515 \mathrm{~nm}, 50 \mathrm{~mW}$, s-polarized \\
\hline & & Jive & $561 \mathrm{~nm}, 50 \mathrm{~mW}$, s-polarized \\
\hline AOTF & AA Opto-Electronic, France & AOTFnC-400.650-TN & $\begin{array}{l}>90 \% \text { diffraction efficiency, 3-nm resolution, low crosstalk } \\
\text { between laser lines, high separation angle }\end{array}$ \\
\hline Laser modulator & Qioptiq GmbH, Germany & LM 0202 VIS ADP & 400 to $650 \mathrm{~nm}, \lambda / 2$-voltage $(633 \mathrm{~nm}): 210 \mathrm{~V}$ \\
\hline Pulse amplifier & Falco Systems, The Netherlands & WMA-300 & $\begin{array}{l}50 \times \text { amplification up to } \pm 150 \mathrm{~V}, \mathrm{DC} \text { to } 5-\mathrm{MHz} \text { signal } \\
\text { bandwidth }\end{array}$ \\
\hline Galvo scanner & Cambridge Technology, United States & $6220 \mathrm{H}$ & Small angle step response $200 \mu \mathrm{s}$ \\
\hline \multirow[t]{2}{*}{ Objectives } & Nikon, Japan & Plan Fluor EPI & $10 \times 0.3 \mathrm{NA}$, WD $17.5 \mathrm{~mm}$, EFL $20 \mathrm{~mm}$ (excitation) \\
\hline & Olympus, Japan & LMPLFLN20X & $20 \times 0.4 \mathrm{NA}$, WD $12 \mathrm{~mm}$, EFL $9 \mathrm{~mm}$ (detection) \\
\hline \multirow[t]{3}{*}{ Motor stages } & Physik Instrumente, Germany & C-863.11 & DC servo-motor controller \\
\hline & & M-122 & $\begin{array}{l}\text { Travel range } 25 \mathrm{~mm}, 0.1 \mu \mathrm{m} \text { resolution, max. velocity } \\
20 \mathrm{~mm} / \mathrm{s}\end{array}$ \\
\hline & & $M-116$ & Precision Rotation Stage, $2.5 \mu \mathrm{rad}$, max. velocity $20 \mathrm{deg} / \mathrm{s}$ \\
\hline Camera & Hamamatsu, Japan & Orca Flash 4.0 v2.0 & $\begin{array}{l}\text { sCMOS sensor, } 2048(\mathrm{H}) \times 2048(\mathrm{~V}) \text {, cell dim.: } 6.5 \mu \mathrm{m} \text {, } \\
\text { active area: } 13.3 \mathrm{~mm} \times 13.3 \mathrm{~mm}, 16 \text { bit images }\end{array}$ \\
\hline DAQ board & National Instruments, United States & NI PCle-6353 & $\begin{array}{l}\text { Al: } 1 \mathrm{MS} / \mathrm{s} \text { multichannel; } 16-\text { bit resolution, } 10 \mathrm{~V} ; \mathrm{AO} \text { : } \\
2.86 \mathrm{MS} / \mathrm{s}, 16 \text {-bit resolution, } 10 \mathrm{~V} \text {; digital I/O lines } \\
\text { (hardware-timed up to } 10 \mathrm{MHz} \text { ), } 100 \mathrm{MHz} \text { max counter } \\
\text { frequency }\end{array}$ \\
\hline Workstation 1 & Dell, United States & T7500 & $\begin{array}{l}12 \text { GB RAM, Intel Xeon Processor X5647 at } 2.93 \text { GHz, } \\
\text { OS Windows } 764 \text { bit }\end{array}$ \\
\hline Workstation 2 & Dell, United States & T5600 & $\begin{array}{l}8 \text { GB RAM, Intel Xeon Processor E5-2620 at } 2 \text { GHz, } \\
\text { OS Windows } 764 \text { bit }\end{array}$ \\
\hline
\end{tabular}

\subsection{Illumination Unit}

The light impinges onto a polarizing beam splitter cube, which splits the excitation light depending on its polarization into one of the two identical excitation arms. The beam diameter is reduced with a telescope ( $f 350$ and $f 200$ ), whose telecentric plane coincides with the mirrored surface of a galvanometric scanner (galvo). Both galvos are mounted on a custom-made optical breadboard, which features two circular holes to pass the periscopic beams and a large central cut-out for the sample chamber and motor stages [Fig. 1(b)].

Each scan mirror surface is reimaged with a telescope ( $f 50$ and $f 100)$ onto the back aperture of a long working distance and low-magnification objective (Nikon, $10 \times 0.3$ NA WD $17.5 \mathrm{~mm}$ ). The focal lengths of the doublets comprising the telescope have been chosen for space considerations and to achieve a $1 / e^{2}$ beam radius of $0.73 \mathrm{~mm}$ at the back focal plane resulting in an effective NA of 0.025 (see also Sec. 2.5). The galvos are in planes conjugated with the back focal plane of their respective excitation objective to ensure that a tilt angle of the galvo converts into a lateral translation of the Gaussian beam across the specimen. The two excitation objectives are designed for air immersion but are immersed into the clearing solution $(n=1.45)$ within the sample chamber. A coverslip glued to the front housing edge serves the dual purpose of maintaining the first diffractive surface between the front optical element and the air and to protect the front lens elements from the clearing agent. A guide to align objectives to a common focus can be found in the Appendix A. The light sheet is generated digitally ${ }^{10,11}$ by scanning the excitation beam across the focal plane of the detection objective. This generates incoherent illumination resulting in fewer artefacts. Additionally, each line in the specimen is illuminated with the same intensity creating a homogeneous light sheet, which is particularly advantageous for the quantitative and qualitative investigation of large samples in their entirety. 


\subsection{Detection Unit}

Fluorescence is collected with an objective that is specifically designed for immersion in clearing solutions. The objective is equipped with a correction collar allowing for immersion in media with refractive indices ranging from 1.41 to 1.52 (Olympus, XLSLPLN25XGMP, 25×, 1.0 NA, effective focal length $7.2 \mathrm{~mm}$, working distance $8 \mathrm{~mm}$ ). A tube lens of $200 \mathrm{~mm}$ creates an image on a sCMOS camera (Orca Flash4.0, Hamamatsu) with a chip of over $4 \mathrm{MP}$. The cell size of the OrcaFlash is $p^{2}=(6.5 \mu \mathrm{m})^{2}$ over an active area of $r^{2}=(13.3 \mathrm{~mm})^{2}$. With a $200-\mathrm{mm}$ tube lens, the field of view (FOV) in the sample is $480 \mu \mathrm{m}^{2}$ (see also Table 2 for relevant formulas).

In the camera's rolling shutter acquisition mode, only a subset of adjacent horizontal pixel lines is simultaneously exposed and this active detection region is moved across the image sensor. ${ }^{12}$ With the delay between the exposure of two adjacent lines set to the minimum time required to read out a single line $\left(t_{\text {shutter }}=\right.$ $9.7 \mu \mathrm{s}$ ), the entire frame of 2048 horizontal lines is activated within $19.86 \mathrm{~ms}$. Setting the line exposure time to $t_{\exp }=$ $0.3 \mathrm{~ms}$, for instance, results in $l=t_{\exp } / t_{\text {shutter }} \approx 31$ lines being simultaneously exposed at any time. This detection area is swept from the top to the bottom of the chip and acts like a moving,

Table 2 Overview of the main formulas related to light-sheet microscopy. See Appendix E for text.

General formulas for microscope objectives

\begin{tabular}{lc}
\hline $\begin{array}{l}\text { Nominal focal length } \\
\text { Effective focal length }\end{array}$ & $f=f_{\mathrm{TL}}^{\text {design }} / \mathrm{M}$ \\
Effective magnification & $f^{\text {eff }}=n f$ \\
Excitation (Gaussian) & $\mathrm{M}^{\mathrm{eff}}=f_{\mathrm{TL}}^{\text {real }} / f^{\text {eff }}$ \\
Beam waist & \\
Axial resolution & $w_{0}=\lambda f_{\mathrm{i}}^{\text {eff }} / \pi W$ \\
Confocal parameter & $\mathrm{FWHM}$ axial $\approx 1.17 w_{0}$ \\
& $\mathrm{~B}=2 \pi \omega_{0}^{2} / \lambda$ \\
& $=2 \lambda\left(f_{\mathrm{i}}^{\mathrm{eff}}\right)^{2} / \pi w^{2}$
\end{tabular}

Detection

Radial resolution

$$
\mathrm{FWHM}_{\text {radial }}=0.5 \lambda_{0} / \mathrm{NA}_{d}
$$

Objective field size

$$
\mathrm{S}=\mathrm{FN} / \mathrm{M}
$$

Camera

Pixel size object space

$$
p_{\text {object }}=p / \mathrm{M}^{\text {eff }}
$$

Camera field of view (FOV) object space

$$
\mathrm{FOV}_{\text {object }}=r_{\text {chip }} / \mathrm{M}^{\text {eff }}
$$$$
=p_{\text {object }} \cdot \#_{\text {pixels }}
$$

Slit width on camera

Slit width in object space

$$
s_{\text {camera }}=p \cdot t_{\text {exp }} / t_{\text {shutter }}
$$$$
s_{\text {object }}=s_{\text {camera }} / \mathrm{M}^{\text {eff }}
$$

Frame rate $\nu=1 /\left(\#_{\text {horiz.lines }} \cdot t_{\text {shutter }}+t_{\exp }\right)$

Note: FWHM, full-width at half-maximum. virtual confocal slit corresponding to a width of $s_{\text {object }}=$ $l p / M_{\text {eff }}=7.2 \mu \mathrm{m}$ in the sample space, where $M_{\text {eff }}$ is the effective magnification of the detection objective. With the given values of line exposure and line readout time, the camera acquires images at a full-frame rate of $R_{\text {frame }}=1 /\left(\#_{\text {horiz.lines }} * t_{\text {shutter }}+t_{\text {exp }}\right) \approx$ $50 \mathrm{~Hz}$.

The camera was run as master in the internal trigger mode and a synchronization pulse generated with the exposure of each new line was used as an output trigger for a DAQ board (NI PCIe-6353, National Instruments), which was operated as slave. The saw-tooth driving signal for the galvo mirrors was generated by the DAQ and synchronized with the pixel line reset pulses from the camera to achieve confocal line detection (see also Sec. 3.2 on synchronization).

\subsection{Sample Mounting and Motion}

We designed a cubic water-tight sample chamber [Fig. 2(a)] that allows access from all six sides while maintaining the 3-D integrity of large clarified and fluorescently labeled mouse brains. The sample chamber is tightly bolted to the optical breadboard while soft connections using silicone bellows allow for adjustive movements of the objectives and free 3-D motion of the motor stages [Fig. 2(b)]. Connections are sealed with rubber rings and silicone caulk and additionally tightened with cable binders. In this way, the objectives can be refocused and realigned without compromising the watertight seal of the chamber [Fig. 2(c)]. The clarified brains are imaged and immersed in clearing solution composed of $63 \%$ 2,2'-thiodiethanol (TDE) in phosphate-buffered saline (PBS) and a refractive index of $n \approx 1.45 .{ }^{13}$ To fill the entire volume of the sample chamber, $\sim 150 \mathrm{ml}$ clearing solution is needed. A motorized $x-, y-, z-, \theta$-stage (see Table 1 ) allows free 3-D motion and rotation of a Teflon cylinder, which reaches into the centre of the chamber [Fig. 2(d)]. Illumination and detection axes are horizontal, whereas sample rotation occurs around the vertical axis.

To elucidate neuronal projections in structurally intact tissue, it is paramount to be able to image centimeter-sized clarified samples, such as mouse brains, with high resolution in whole mount preparation. The problem of sample mounting in a light-sheet microscope arises from the fact that optical access to the sample is required from three to four planar sides leaving only two opposing sides to insert, fix, and move the sample. Stable mounting is hereby a key concern as a whole-brain tomography can require image acquisition in excess of $24 \mathrm{~h}$ and the effects of gravity, tissue shrinking/expansion and evaporation of the clearing solution over such time spans might have to be considered.

For the acquisition of whole-brain datasets, we fix a clarified and fluorescently labeled mouse brain with super glue to a coverslip [Fig. 2(e)]. The brain is oriented along its axial orientation with the olfactory bulbs at the top and the cerebellum at the bottom of the coverslip. The coverslip is slid into a bottom adaptor and tightened with a plastic-capped grub screw. The bottom adaptor is inserted into a Teflon cylinder with the coverslip being positioned on the far side of the detection objective. Three different slits in the adapter correspond to varying distances to the detection objective and give variability in sample thickness, for example, to allow also for the mounting of rat brain hemispheres. 

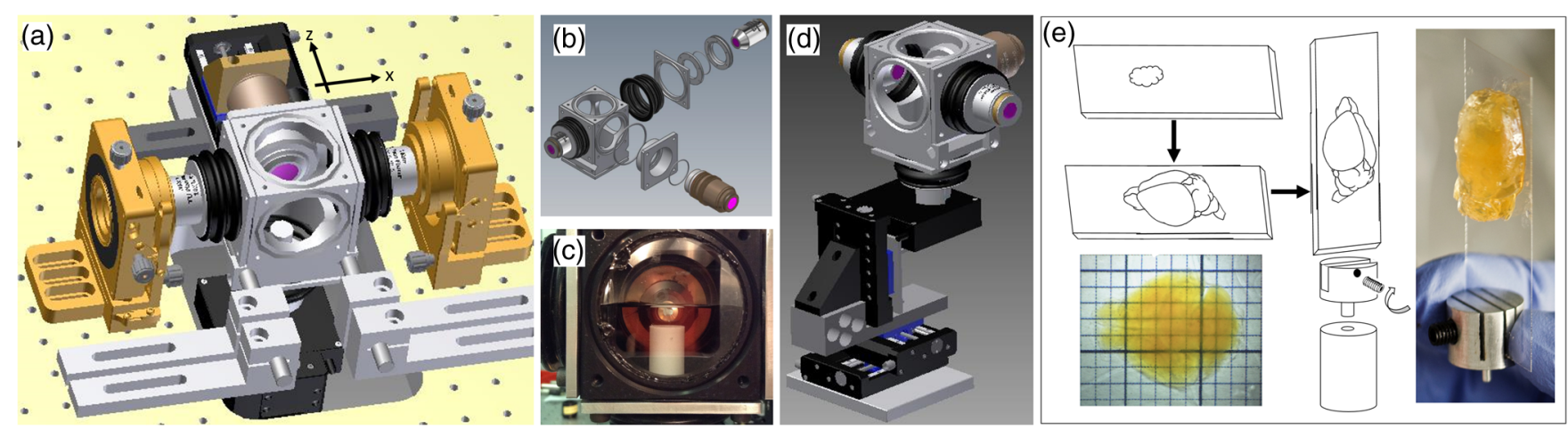

Fig. 2 Schematic of sample mounting and motion. (a) Fluorescence excitation (along $x$ axis) and detection (along $z$ axis) are operated on independent perpendicular light paths where the excitation light sheet and the detection focal plane overlap. (b) The custom-made sample chamber is assembled with silicone bellows and seal rings. The clarified fluorescently labeled brain is mounted on a Teflon cylinder in the center of the watertight chamber filled with clearing solution (c) and can be translated and rotated freely with piezo motors (d). (e) Mounting of a clarified fluorescently labeled brain. The brain is glued onto a coverslip and inserted into an adapter that slides into the Teflon cylinder.

\subsection{Optical Characterization}

\subsubsection{Theoretical considerations}

The optical properties of a microscope can be quantified by the point-spread function (PSF), which is the image of a point source in object space. The radial (i.e., perpendicular to the detection axis) and axial (i.e., parallel to the detection axis) resolution of the microscope are most commonly described by the full-width at half-maximum (FWHM) of the PSF itself along the chosen direction [Fig. 3(a)]. In a light-sheet microscope, axial and radial resolution are determined by the numerical aperture (NA) of the illumination and detection optics, respectively. The radial PSF is a standard Airy function whose FWHM is given by

$\mathrm{FWHM}_{\text {radial }} \simeq \frac{0.51 \lambda_{0}}{\mathrm{NA}_{\mathrm{d}}}$,

where $\lambda_{0}$ is the wavelength in vacuum and $\mathrm{NA}_{\mathrm{d}}$ is the NA of the detection objective. The axial resolution is dominated by the thickness of the light sheet which, for high NA detection objectives, is usually larger than the depth of field of the detection objective. If Gaussian beams are used for illumination, the thickness of the light sheet can be calculated by the well-known formulas of Gaussian optics. ${ }^{14}$ The radius of the beam waist is given by $w_{0}=\frac{\lambda f_{\mathrm{i}}^{\mathrm{eff}}}{\pi w}$,

where $w$ is the $1 / e^{2}$ radius of the beam entering the illumination objective, $f_{\mathrm{i}}^{\text {eff }}$ is the effective focal length of the illumination optics, and $\lambda$ is the wavelength in the medium $\left(\lambda=\lambda_{0} / n, n\right.$ being the refractive index). Note that for air objectives (as the one we use for illumination), the effective focal length is extended by a factor $n$ when used inside a medium with refractive index different from unity. ${ }^{15}$ The axial FWHM is then given by

$\mathrm{FWHM}_{\text {axial }} \simeq 1.177 w_{0}$

The thickness of the light sheet is not constant throughout the FOV due to the limited depth of focus of the Gaussian beam. The latter can be quantified by the confocal parameter $b$, which is the distance along which the beam $1 / e^{2}$ radius remains relatively constant and smaller than $\sqrt{2} w_{0}$ :

$b=\frac{2 \pi w_{0}^{2}}{\lambda}=\frac{2 \lambda\left(f_{\mathrm{i}}^{\mathrm{eff}}\right)^{2}}{\pi w^{2}}$.

The need to have an almost uniform resolution in the camera FOV imposes a lower bound to $b$ and thus (according to the
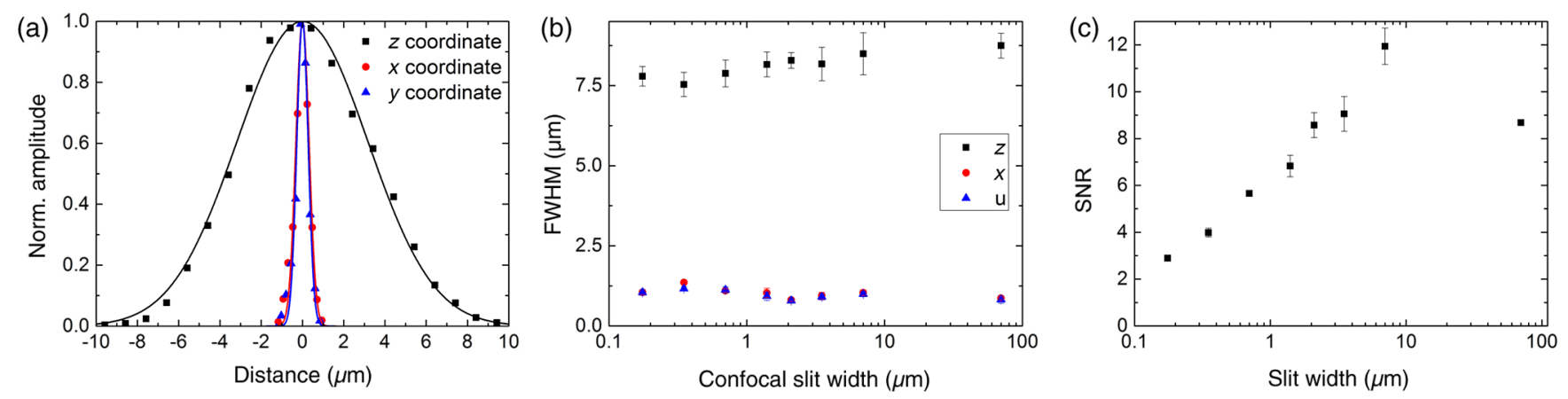

Fig. 3 Optical characterization. (a) Exemplary lateral and axial intensity profiles of nanometric beads and fits to Gaussian curves. (b) Full-width at half-maximum (FWHM) of the Gaussian fits with confocal slit width. Each data point is the average of 10 bead profiles. (c) Signal-to-noise ratio (SNR) as a function of slit width. 
previous equations) a limit to the axial resolution. In our setup, we adjusted the telescopes in the excitation path in order to have $w \simeq 0.73 \mathrm{~mm}$, resulting in $b \simeq 346 \mu \mathrm{m}$ (corresponding to $72 \%$ of the $\mathrm{FOV}$ ) and $\mathrm{FWHM}_{\text {axial }} \simeq 5.13 \mu \mathrm{m}$ (assuming $\lambda_{0}=0.5 \mu \mathrm{m}$ ). An overview of the relevant formulas concerning the optical properties of a light-sheet microscope is given in Table 2 (see Appendix E for text). The reader is referred to this table as a quick guide to tailor their microscope to their specific needs.

\subsubsection{Experimental characterization}

For optimal imaging, it is crucial that the sample-emitted line of fluorescence is in the centre of the rolling shutter at the start of each image acquisition and furthermore travels with the same speed during stack acquisition. To this end, the galvo scanners were calibrated using a fluorescein solution in the sample chamber and an image of the focal line was acquired for a set of mirror angles. By fitting each focal line profile to a Gaussian curve, the position of the beam main axis was determined and plotted against the voltage applied to the galvo scanner. A linear fit to this data yielded the precise amplitude and offset for each galvo scanner. The finite width of the confocal slit was hereby taken into account such that maximum beam deflection corresponded to the central pixel line of the confocal slit coinciding with the first and last pixel row on the camera chip. From the same data, the average FWHM of the Gaussian excitation line over the FOV of the camera was calculated as $(8.60 \pm 0.67) \mu \mathrm{m}$ $(n=14)$. The disagreement with the theoretical value of $5.13 \mu \mathrm{m}$ is likely attributable to the refractive index mismatch introduced in the excitation arm by the protective coverslip and by the optical path inside a medium with a refractive index different from air, both causing spherical aberrations. ${ }^{16}$

For quantitative analysis of spatial resolution and the SNR, image stacks of nanometric fluorescent beads $(0.51 \mu \mathrm{m}$ diameter, Bangs Laboratories) were acquired (see Apendix B for sample preparation). The spatial resolution, in agreement with previous findings, ${ }^{17,18}$ was not affected by the confocal slit width on the camera [Fig. 3(b)]. The lateral and axial
FWHMs were $(1.00 \pm 0.04) \mu \mathrm{m}$ and $(8.13 \pm 0.14) \mu \mathrm{m}$, respectively, averaged through stack depths and confocal slit widths. The experimentally determined lateral FWHM was larger than the theoretically calculated value of $0.26 \mu \mathrm{m}$ by a factor of four. The discrepancy is likely caused by higher-order aberrations introduced by the gel embedding the fluorescent beads exacerbated by the high NA of our detection objective. ${ }^{19,20}$

Qualitatively, the SNR increased with increasing slit width up to a maximum around the $1 / e^{2}$ illumination beam diameter of $8.6 \mu \mathrm{m}$ and then decreased again with further increasing slit width [Fig. 3(c)] in agreement with previous observations. ${ }^{12}$

\section{Microscope Management Software}

\subsection{General Design}

The many components of the microscope (camera, lasers, AOTF, stages, etc.) are orchestrated and operated through a custom-made software written in the LabVIEW language (National Instruments, Austin, United States). Since the microscope should work mainly without human intervention for many hours (a whole-brain tomography can last a couple of days) without losing precise synchronization, stringent software design is a primary concern. The overall architecture of the software and its practical implementation were chosen to satisfy two main principles: first, operation in a distributed environment and second, robustness and ease of maintenance. This means, the apparatus should be able to deal with many independent hardware components and with several data streams (see below). Individual processing steps and/or data transfer can be computationally intensive and may need to be distributed over several computers to perform properly at runtime. Any kind of hardware failure, from timing delays to complete system crash, must be automatically handled to avoid loss of data.

An object-oriented programming framework ${ }^{21}$ has been used for software development, dividing the entire project into well-defined self-contained modules. This framework, besides helping defining the proper scope of each software component, guarantees robustness, ease of maintenance, scalability, and
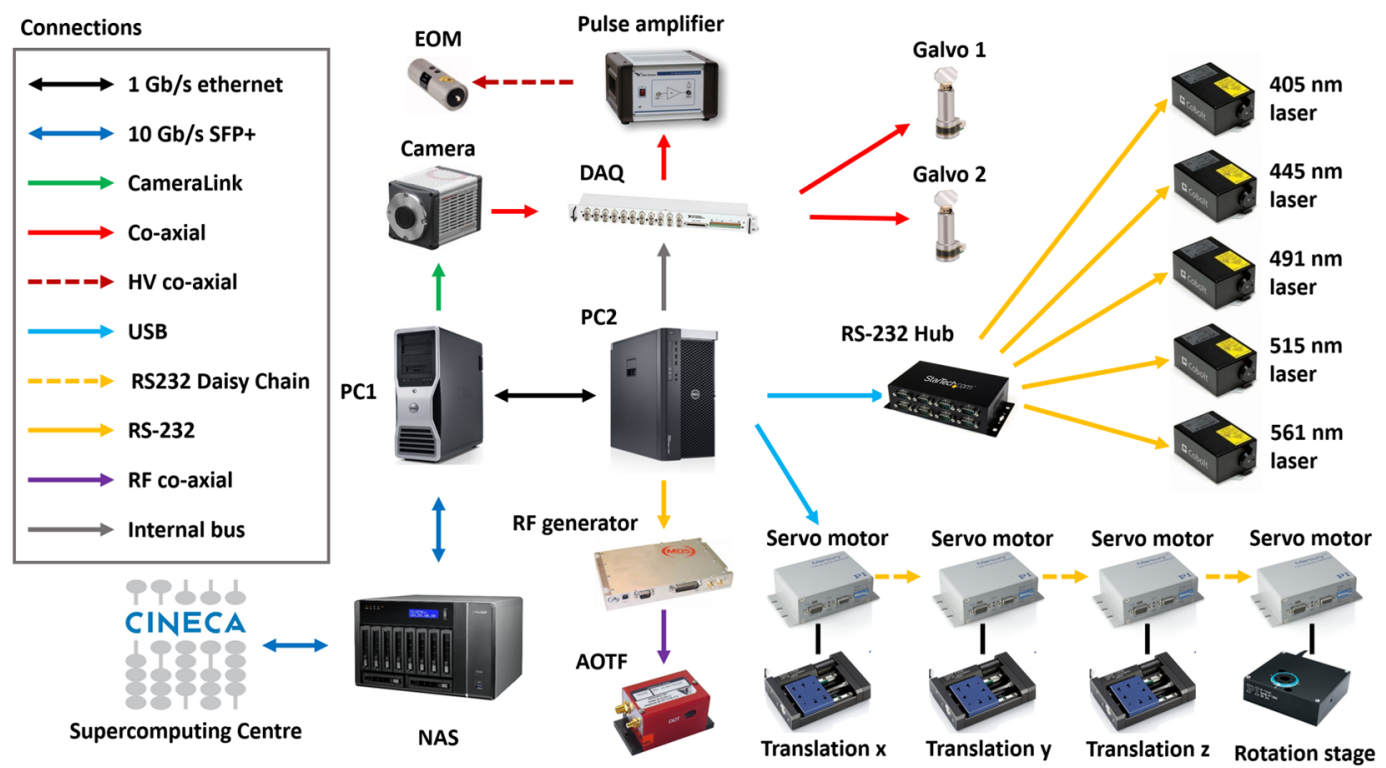

Fig. 4 Schematic view of the hardware architecture of the microscope. Different kinds of physical connections are depicted with different colors. 
reusability. Code was stored on GitHub (www.github.org) to help multiple users developing software simultaneously. The Murmex software development kit (see Appendix D) was used to operate the software efficiently and synchronously on different computers.

\subsection{System Timing}

A schematic overview of the hardware architecture of our LSFM is shown in Fig. 4. One personal computer (Precision T5600, Dell, see also Table 1) controlled all the remote hardware components with the exception of the camera, which was controlled by a second, dedicated computer (Precision T7500, Dell) to handle and manage the enormous streams of data produced (see also Sec. 4). Various instrumentations were addressed either through computer ports (as USB, RS232, and CameraLink) or via analog and/or digital signals generated by the DAQ board.

Synchronization of the camera rolling shutter to the scanning of the light sheet was achieved by letting the camera acquiring in free-run mode and using a trigger output from the camera itself to time the generation of the waveforms sent to the galvo drivers. The HSYNC signal from the camera was used which provided transistor-transistor-logic pulses every time the rolling shutter stepped from one line to the next one. This trigger signal was used as a clock by the DAQ board so that the line producing the light sheet moved step by step with the rolling shutter. The same HSYNC signal was also used to trigger the DAQ digital signal sent to the laser modulator. Since at each position of the scanning line the modulator should go through at least two states (vertical and horizontal polarization), an HSYNC pulse was used as a trigger signal while the much faster internal clock of the DAQ board served as a clock.

\section{Data Management}

The volume of an adult mouse brain has been estimated from MRI measurements ${ }^{22}$ as $\simeq 0.5 \mathrm{~cm}^{3}$; the same authors determined that a parallelepiped encompassing the whole brain would have a volume of $\simeq 0.9 \mathrm{~cm}^{3}$. Given the pixel size of the camera and the magnification of the system, and assuming a $z$ step of $2 \mu \mathrm{m}$, the voxel size amounts to $0.234 \times 0.234 \times 2 \mu \mathrm{m}^{3}$. This means that a mouse brain will be represented with about 4.6 TeraVoxels, whereas a parallelepiped encompassing the brain will result in 8.2 TeraVoxels. Since the camera produces 16 bit images with no compression, each raw voxel occupies 2 bytes of disk space, resulting in $8.3 \mathrm{~TB}$ solely for the whole brain and 14.8 TB for the encompassing parallelepiped (see Table 3). However, image stacks are acquired with $10 \%$ of linear superposition, which is later used for image stitching. This redundancy expands the size of raw data to 10.2 and 18.3 TB, respectively.

This tremendous amount of data challenges the traditional way we interact with our data. Having far passed the point of taking your data home with you on a hard drive at the end of the experiment, the handling of data in light-sheet microscopy requires the development of a robust data management infrastructure and of novel software tools to process the images in order to extract meaningful information.

The camera flushed the data stream directly to a dedicated workstation with 12 solid-state drives (SSD) operating in RAID 0 configuration, resulting in a virtual drive of 10.9 TB. Although this was already a large and expensive system for the current SSD technology, it was much smaller than the size of the raw datasets produced in each tomography. We

Table 3 Data production rates for typical camera operation.

Voxel size

Frame rate

Frame size

Frame disk size

Data production rate

Data conversion rate

Net solid-state drives (SSD) filling rate

SSD RAID size

Max acquisition time to fill up SSDs

Max raw data size that can be acquired continuously

Max volume that can be imaged (considering $10 \%$ superposition between adjacent stacks)

thus connected the workstation to a larger network area storage (NAS) via a $10 \mathrm{Gbit} / \mathrm{s}$ network copper cable. The NAS operates in a safer data modality (RAID 5) with 32.4 TB of useful disk space. The NAS is then connected by a dedicated 10 Gbit/s link provided by the Consortium GARR (the public institution managing research networks in Italy) to CINECA (the main Italian supercomputing center). The data flow scheme is summarized in Fig. 5.

While transferring data from the SSDs to the NAS, we perform on-the-fly conversion of files generated during image acquisition to a losslessly-compressed, multipage TIFF format. Our conversion module leverages the functionalities offered by the interface of the DCIMGAPI library, supplied by Hamamatsu, and the freely available LibTIFF library. The module reads a single .cxd 3-D image and writes it in a variable number of multipage TIFF files, depending on the user-defined limit of pages per file. Since the output of the Hamamatsu camera may exceed tens of gigabytes, the image in proprietary format is read slice by slice to minimise memory requirements. The conversion module runs concurrently with the microscope controlling software and is synchronized to convert stack files as soon as they are completed. The module provides options to downsample the image or to save the input image at full or reduced resolution in order to adapt to storage requirements. Usually, we downsample the image in the $x y$ plane by a factor 2 and leave the $z$ sampling unaltered. This downsampling, in combination with the lossless compression offered by the TIFF format, reduces data size by about 1 order of magnitude. Thus, the parallelepiped volume containing the whole mouse brain takes up about 2 to 3 TB of disk space.

The process executing the file conversion was set to low priority to prevent interference with the camera component, which needs to be perfectly timed with acquisition. Although file conversion drastically reduces data size, in the current implementation, it was slower than data production. The camera flushed data to the SSDs with a rate $R_{\text {data }}$ dependent on the frame rate $R_{\text {frame }}$ and on the image size: $R_{\text {data }}[$ Bytes $]=2 \cdot R_{\text {frame }} \cdot \#_{\text {pixels }}$, where the factor of 2 takes into account the 16 bit grey 

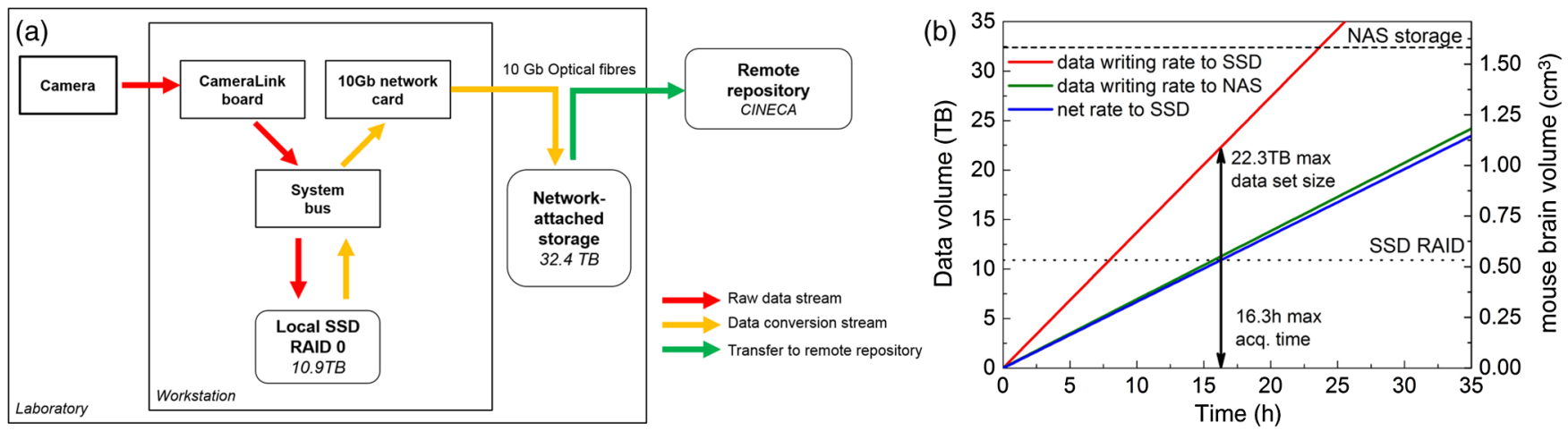

Fig. 5 Scheme of the data flow. (a) Data streaming on the microscope. SSD: solid-state disk, RAID: redundant array of independent disks. Colors indicate the time-criticality (red: higher to green: lower) of each data stream. (b) Data production rate versus data conversion rate showing the maximum acquisition time and tomography size feasible before the SSD are filled up. NAS: network-attached storage.

depth of images. The volumetric imaging rate is given by $R_{\text {volume }}=0.5 R_{\text {data }} \cdot$ voxelsize.

With a standard image size of $2048 \times 2048$ pixels, and $R_{\text {frame }}=50 \mathrm{~Hz}$, data were produced at a rate $R_{\text {data }}=$ $400 \mathrm{MB} / \mathrm{s} \approx 1.37 \mathrm{~TB} / \mathrm{h}$, which resulted in a volumetric rate $R_{\text {volume }} \approx 2.3 \times 10^{7} \mu \mathrm{m}^{3} / \mathrm{s} \approx 0.08 \mathrm{~cm}^{3} / \mathrm{h}$. The conversion module freed the SSDs with an average rate of $\approx 0.7 \mathrm{~TB} / \mathrm{h}$, meaning that the SSDs were actually filled up during imaging at a rate of $\approx 0.67 \mathrm{~TB} / \mathrm{h}$. Imaging sessions needed thus to be shorter than $16.3 \mathrm{~h}$, which limited the maximum amount of raw data being acquired continuously to $\approx 22.3 \mathrm{~TB}$. However, this was well above the disk space needed to image the parallelepiped volume encompassing a whole mouse brain. Larger specimens, as rat brain or portions of human brain, must be imaged in consecutive session until faster strategies for image conversion are devised.

After acquisition, the tiled raw images produced had to be stitched to a 3-D dataset. To perform this operation, we used the TeraStitcher, ${ }^{23}$ a fully automated stitching tool designed to deal with tiled images of virtually unlimited size. TeraStitcher can run on machines with limited resources with a performance essentially determined only by the transfer rate toward secondary storage. TeraStitcher can be freely downloaded from Ref. 24.

\section{Representative Results}

Some representative data produced by our microscope are reported in Fig. 6. A detailed description of sample preparation can be found in Appendix C. With the apparatus described in this paper, whole mouse brains could be imaged in their entirety [Fig. 6(a)], maintaining a high quality of the image also when going deep inside the specimen [Fig. 6(b)]. The resolution and contrast of the images allowed to clearly distinguish axonal tracts [Fig. 6(c)] in the brain of a PV-cre-dTomato transgenic mouse, in which parvalbuminergic neurons are fluorescently labeled. Lateral and axial intensity profiles through a fine axonal projection were used to estimate resolution in a brain dataset in terms of the FWHM in the lateral and axial directions [Fig. 6(d)]. The lateral and axial FWHM were 1.7 and $8.5 \mu \mathrm{m}$, respectively, in agreement with the measurements on fluorescent beads. Whole-brain datasets, as presented here, can be used to localize cell distributions in 3-D using automatic cell-detection software. ${ }^{7}$
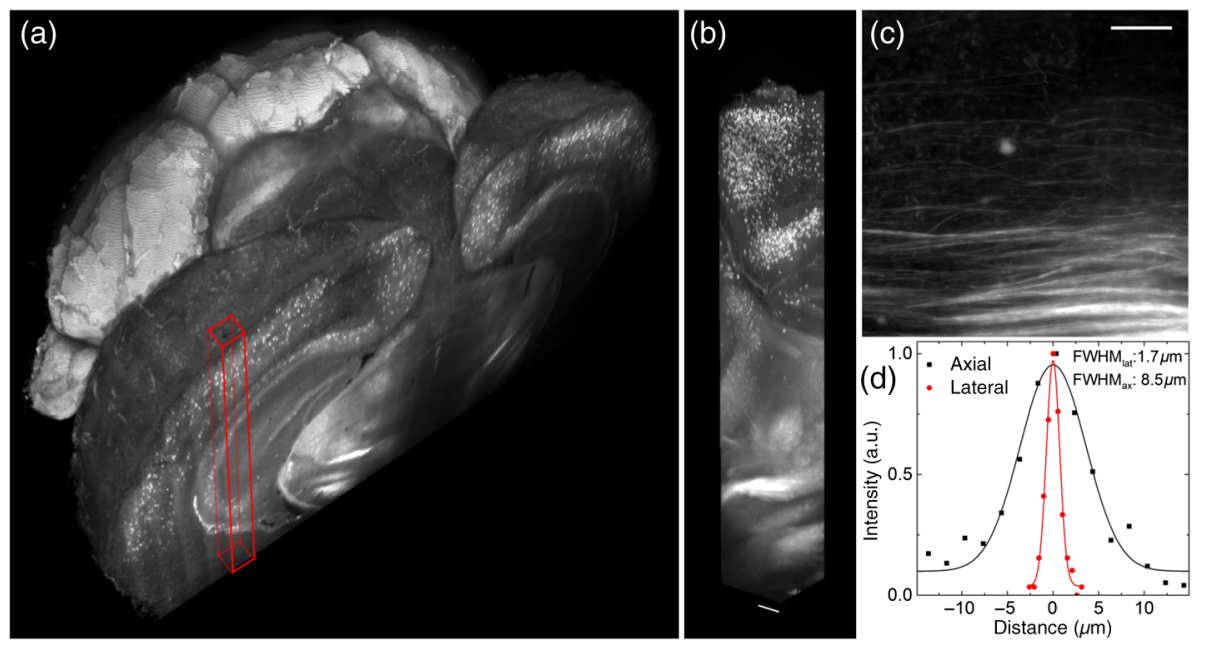

Fig. 6 Whole mouse brain tomography. Imaging of whole transgenic mouse brain treated with CLARITY and cleared with $2,2^{\prime}$-thiodiethanol $63 \%$ imaged with an Olympus, $25 \times$ objective. (a) $3-D$ rendering of a parvalbumin-dTomato brain. (b) 3-D rendering of a stack from PV-dTomato mouse brain, scale bar $=400 \mu \mathrm{m}$. (c) High resolution insert showing axonal projections. Scalebar $=100 \mu \mathrm{m}$. (d) Lateral and axial FWHM of the intensity profiles through single axon. Modified with permission from Ref. 13 


\section{Discussion and Conclusion}

Light-sheet microscopy has already been a game changer for large-scale, high-throughput imaging by yielding data of high spatiotemporal scale. This measurement technique will continue to have a high impact in the field of whole-brain imaging due to its ability to record millions of images over the course of days or even weeks. Here, we have presented a complete and detailed framework for whole mouse brain imaging. Starting with a full description of our double-sided light-sheet microscope and its constituent components, we further gave details of the custom-written control software, which coordinates the microscope's reliably synchronized operation. The data produced in our experiments easily amounts several TB per data set and needs to be compressed, stored, transferred, retrieved, and processed necessitating the concurrent development of novel computational interface and analysis methods. Here, we have presented a comprehensive, robust, and fully automated pipeline of data management starting from the streaming of raw images up to the stitching of 3-D datasets.

The mouse brains imaged in our microscope are intrinsically opaque and need to be clarified before imaging. A successful optical clearing method not only renders the tissue transparent but also causes neither quenching of protein fluorescence nor tissue distortion and is compatible with repetitive immunostaining. A very promising method called CLARITY ${ }^{8,9}$ transforms tissue into a nanoporous, hydrogel-hybridized, lipid-free form. Crucially for the high-throughput imaging of large samples that we aim for, the protocol, however, is also fast, easy, and cheap. We therefore, developed a versatile, simple, rapid, and inexpensive clearing method described elsewhere, ${ }^{13}$ which in combination with CLARITY allows for the optical clearing of entire mouse brains or portions of human tissue.

Even in refractive index-matched samples, we have noticed that significant aberrations degrade image quality, especially at depths toward the end of the stack. Recently, objectives specifically conceived for LSFM have become commercially available, ${ }^{25}$ which have been designed for use with clearing solutions and typically also feature a correction collar for the compensation of a certain amount of spherical aberration. A complete correction of system-induced aberrations, however, requires the ability to also correct for residual spherical aberration and higher-order aberrations introduced by the sample mount, immersion medium, and slight misalignments of the optical system. Of course, aberrations introduced by the sample itself also degrade image quality and it is those aberrations that are most difficult to correct at runtime due to their modal complexity, large amplitude, and high spatial variability. ${ }^{26}$

Additionally, another problem inherent to light-sheet microscopy degrades image quality due to the uncoupled nature of excitation and detection in LSFM, the aberrations encountered in each arm are independent too and this can lead to a walkoff between the light sheet and the detection FOV when large samples are imaged at depths. Adaptive optics ${ }^{27}$ provides a means to compensate for these aberrations and has recently been applied to improve LSFM. $^{28}$ The prospect of high quality images is especially essential for high throughput imaging methods for whole-brain 3-D reconstructions because the size of datasets is so large that they warrant fully automated postprocessing, for example, for automated cell counting and identification. ${ }^{7}$ The effect of optical aberrations can severely obscure features even above the resolution limit and therefore hinder automated segmentation of the data. We have mounted the camera on a separate breadboard, which leaves us with much free, infinity-corrected space in the detection path. Implementation of adaptive optics technology into the fluorescence emission path of our LSFM in order to demonstrate real-time aberration corrected images for whole-brain reconstructions will be considered in the future.

Further improvements in image quality may be achieved with beam shaping in the illumination arm, for example, by implementing Bessel beam illumination. ${ }^{29-31}$ Using these self-reconstructing, nondiffractive beams, the optical sectioning capability can be improved over an extended FOV.

The streamlining of high-throughput imaging of entire mouse brains, starting from the live animal up to quantifiable datasets, requires tight integration of all aspects of the work flow. Even if the brains we image are transparent, their large size means that also comparatively small heterogenities in the refractive index matching sum up to large optical path differences and therefore strong aberrations at large imaging depth. The microscope used for whole-brain imaging needs to be developed and adapted bearing those imaging circumstances in mind and sample preparation methods, imaging technology, and data management schemes must work as a unit in order to deliver on the highly anticipated, quantifiable datasets of whole-brain neuronal projections.

\section{Appendix A: Alignment}

For LSFM, it is crucial that excitation and detection occur on perpendicular axes because any deviation from this geometry results in obscured images of reduced resolution and contrast. The objectives need to be perfectly confocal such that the fluorescence generated in the swept excitation beam also falls within the detection objective's focal plane. Telecentric imaging, that is, the imaging with two lenses, which are the sum of their focal lengths apart [Fig. 7(a)], is used to reimage the galvo scanners onto the back apertures of the excitation objectives. In the detection path, the camera chip needs to be positioned in an image plane of the detection objective. Additionally, homogeneous illumination from both sides imposes strict symmetry considerations on both illumination arms that have not only to be sufficiently aligned with respect to themselves but function as a pair with recursive dependence.

Our microscope was aligned with two tools, a shear plate to qualitatively assess collimation and a small mirror that can be mounted inside the sample chamber. The 0.5 -in. mirror was first pierced with a drill using a ceramic drill bit to produce a hole roughly in its centre of the approximately the same diameter as the excitation beam inside the sample chamber [Fig. 7(b)]. Using a compact single axis, adjustable mirror mount (V50AX, Newport) attached to the Teflon cylinder allows to adjust the pitch of the reflection with the mount and the yaw with the rotation stage while at the same time providing a very space efficient mounting for the pierced 0.5 -in. mirror. With this "sample mirror," three different positions can be easily implemented: first, back reflection by hitting the reflective surface at 0 deg; second, transmission by laterally displacing the mirror until the light passes through the drilled hole [Fig. 7(c)]; and third, reflection of the light by 90 deg by precisely turning the mirror mount by $45 \mathrm{deg}$ using the rotation stage [Fig. 7(d)].

The excitation objectives are fixed on mounts, which allow 3-D translation plus pitch and yaw adjustment (LP-1A, Newport). In a first alignment step, the beam paths of both 

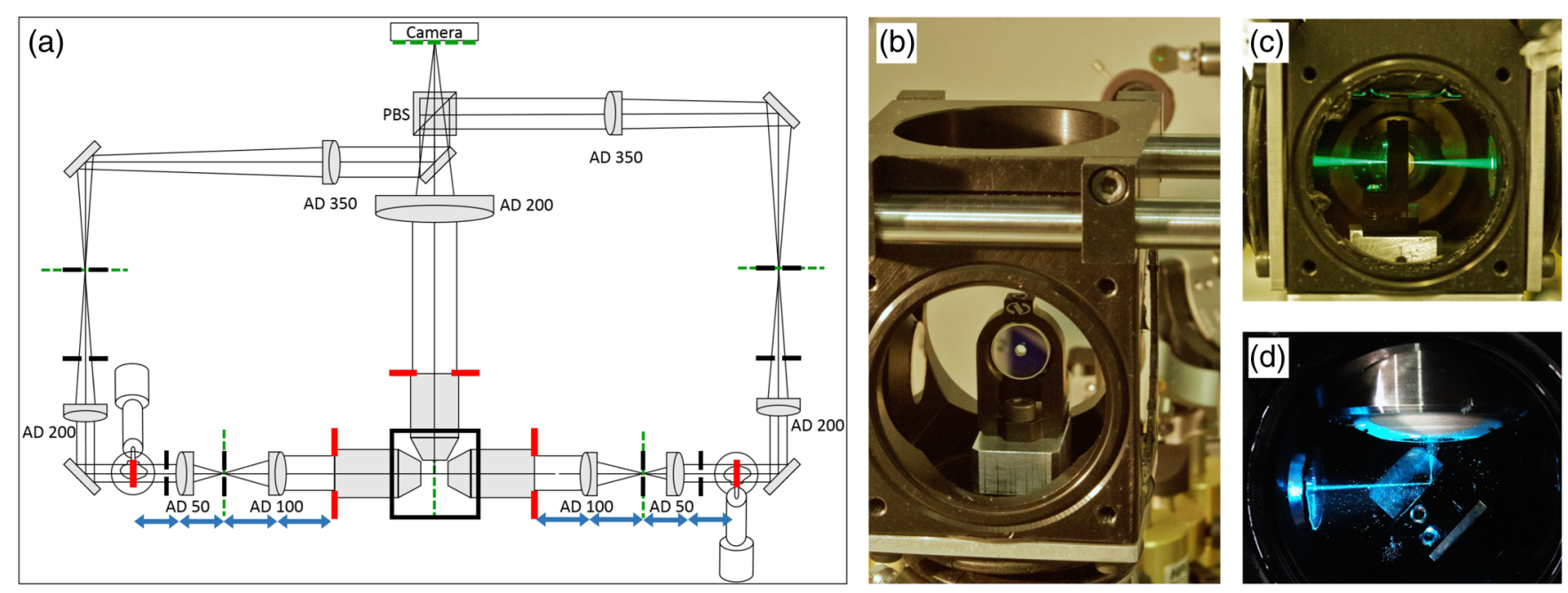

Fig. 7 (a) Basic geometrical optics of a double-sided illumination light-sheet microscope. AD: achromatic doublet, PBS: polarizing beam splitter, red: objective back focal planes and conjugated telecentric planes, green: image planes, blue: $4 f$ telecentric lens system, black: alignment irises. (b) Alignment mirror with drilled hole for light transmission, mount for tip adjustment and adaptor for the Teflon tube. (c) With lateral movement the alignment mirror can be placed such that light is transmitted into the opposing excitation arm. (d) The alignment mirror can be rotated to precisely reflect by 90 deg.

excitation arms were brought to overlap through irises placed on the breadboard and the optical bench without any microscope objectives. We found it useful to use two mirrors in each periscope: one vertically mounted, and one mounted at 45 deg for vertical deflection of the incoming beam. In this way, full beam steering can be achieved to realign the periscope before hitting the galvo scanner. After this initial alignment, the sample mirror was placed with its reflective surface in the centre of the central cut-out of the breadboard. The first excitation objective was placed into the beam path and brought to focus onto the sample mirror, which was adjusted to reflect the light back into the same objective through the irises. Using a shear plate and a beam splitter cube, the back reflected light was qualitatively adjusted for collimation using translation along the excitation axis of the objective mount [Fig. 8(b)]. The sample mirror was then moved laterally to allow the excitation beam to pass through the drilled hole and the second excitation objective was placed [Fig. 8(c)].
This time collimation was checked with the light going through both objectives and so their confocal placement was ensured. Both excitation arms were then aligned recursively by starting at the putative confocal point between the objectives and placing successively lens after lens in the direction toward the light source, alternating between the two modalities illustrated in Fig. 8(a). Finally, the sample mirror was positioned in deflection mode and the detection objective was aligned [Fig. 8(d)].

\section{Appendix B: Sample Preparation for Optical Characterization}

The fluorescent beads (CP01F, Bang Laboratories, $0.51 \mu \mathrm{m}$ diameter) used for quantitative analysis of spatial resolution and SNR were embedded in low-melting point agarose (4\% agarose in $63 \%$ TDE in PBS). The agarose (A9793, SigmaAldrich) was dissolved and then mixed with a diluted bead
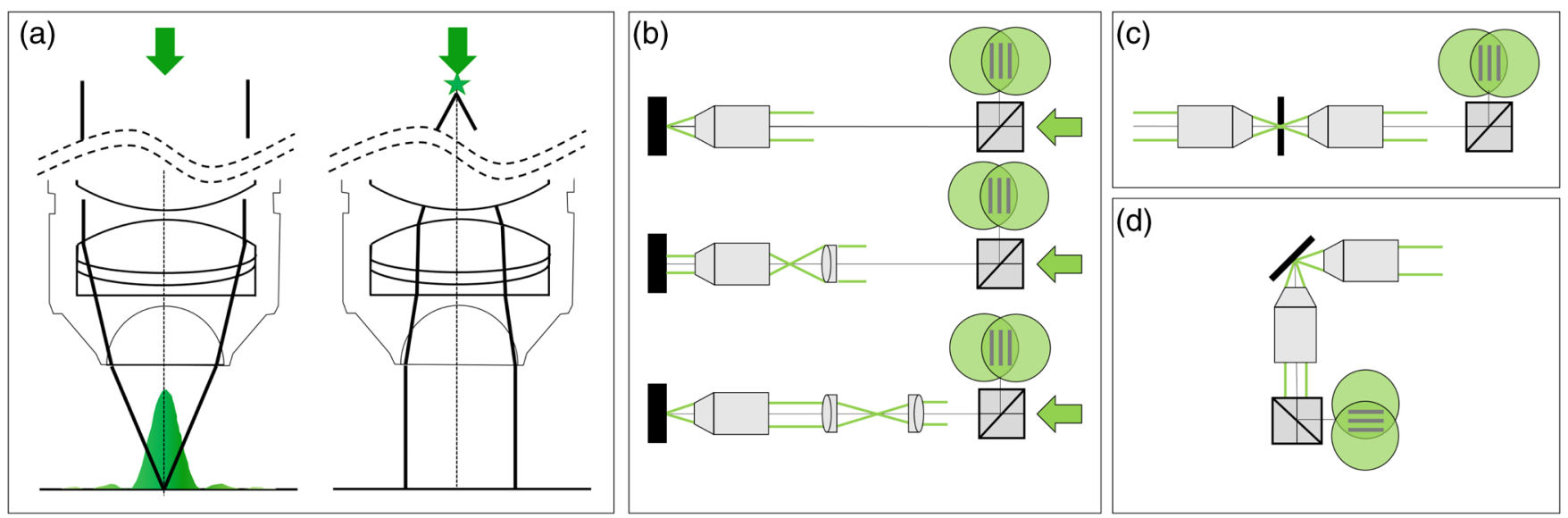

Fig. 8 (a) Working principle of a microscope objective (left): a collimated input beam is converted into a spherical wavefront. Right: inverted light path, a focal spot in the back aperture creates a collimated output. (b) Recursive placement of lenses using the sample mirror in back-reflection mode, (c) in transmission mode, and (d) in reflection mode. 
solution and left to polymerize over night on a coverslip. The coverslip was mounted inside the sample chamber as described in Sec. 2.4. The embedded subdiffraction fluorescent beads were imaged with varying confocal slit widths ranging from 0.175 to $70 \mu \mathrm{m}$ in the sample space. For each slit width, one stack of $200 \mu \mathrm{m}$ depth ( $1 \mu \mathrm{m}$ step size $)$ was acquired and the one-dimensional PSF in the lateral and axial directions was extracted manually with FIJI/ImageJ throughout the depth of the image stack. After fitting the lateral and axial profiles with Gaussian curves, the lateral and axial FWHM were averaged ( $n=10$ data points) for different slit widths. The SNR was calculated according to $\mathrm{SNR}=\left(y_{0}+A\right) / y_{0}$, where $y_{0}$ and $A$ are the offset and amplitude of the Gaussian fits.

\section{Appendix C: Sample Preparation for Whole-Brain Imaging}

Mouse brains were processed using a modified CLARITY protocol $^{8}$ and cleared with a new refractive-index-matching medium. ${ }^{13}$ Adult mice (postnatal day 56) were anaesthetized with isoflurane and transcardially perfused with $20 \mathrm{ml}$ icecold PBS solution ( $\mathrm{pH}$ 7.6) followed by $20 \mathrm{ml}$ of a mixture of $4 \%$ (wt/vol) paraformaldehyde (PFA), 4\% (wt/vol) acrylamide, $0.05 \%$ (wt/vol) bis-acrylamide, $0.25 \%$ (wt/vol) VA044 in PBS. We then extracted the brains from the skull and put them in the same PFA/acrylamide/bis-acrylamide/VA044/PBS solution at $4^{\circ} \mathrm{C}$ for 3 days. Afterward, we degassed the samples and increased their temperature to $37^{\circ} \mathrm{C}$ to initiate polymerization. The embedded samples were then extracted from the gel and washed with clearing solution solution [sodium borate buffer (200 mM, pH 8.5) containing 4\% (wt/vol) sodium dodecyl sulfate] at $37^{\circ} \mathrm{C}$ through gentle shaking. To perform electrophoretic tissue clearing (ETC), hydrogel-embedded brains were placed in a custom-built organ-electrophoresis chamber. The same clearing solution was circulated through the chamber and a voltage of $20 \mathrm{~V}$ was applied across the ETC chamber at $37^{\circ} \mathrm{C}$ for several days. After clearing, brains were incubated in PBST0.1 (PBS and $0.1 \%$ Triton X-100, $\mathrm{pH} 7.6$ ) at $37^{\circ} \mathrm{C}$ for 2 days to remove the clearing solution. As a final step, murine brains were incubated in $50 \mathrm{ml}$ of
$30 \%$ and $63 \%$ (vol/vol) 2,2'-thiodiethanol in $0.01 \mathrm{M}$ PBS (TDE/PBS), each for 1 day, respectively, at $37^{\circ} \mathrm{C}$ while gently shaking. The final $63 \% \mathrm{TDE} / \mathrm{PBS}$ solution has a refractive index $n \simeq 1.45$, allowing for refractive index matching and thus tissue transparency. The experimental protocols involving animals were designed in accordance with the laws of the Italian Ministry of Health. All experimental protocols were approved by the Italian Ministry of Health.

\section{Appendix D: Murmex}

To operate the software efficiently and synchronously on different machines, the Murmex software development $\mathrm{kit}^{32}$ was used. This kit integrates the object-oriented programming paradigm with a messaging system and can be used to create communicating yet independent software modules within a standardized programming scheme.

Murmex creates distributed finite state machines, design patterns in which one component proceeds from a particular state to another based on the message it receives either from itself or from other components. For sending and receiving messages, Murmex uses the library LabbitMQ ${ }^{33}$, which is a wrapper of RabbitMQ ${ }^{34}$, a message-oriented middle-ware; however, these layers of abstraction are mostly hidden from the developers. Buffered, asynchronous, and reliable communication between several software components is established by routing of messages by the broker to the specific software component pinpointed by its ID. Murmex has 12 predefined states (e.g., Initialize, Configure, Start, Stop, Proceed, Update) and a generic state for implementing custom states. Usually, just a few of this states are actually used in standard applications. An example of a standard implementation of the Murmex state machine for the camera is shown in Fig. 9. Each Murmex component streams information about its current status to its "observers," which are other components specified in its configuration file. Using this oberserver/observee hierarchy, it is possible to design a large and complex network of different components, running on various computers connected through a local connection or the internet while maintaining precise timing and synchronization.

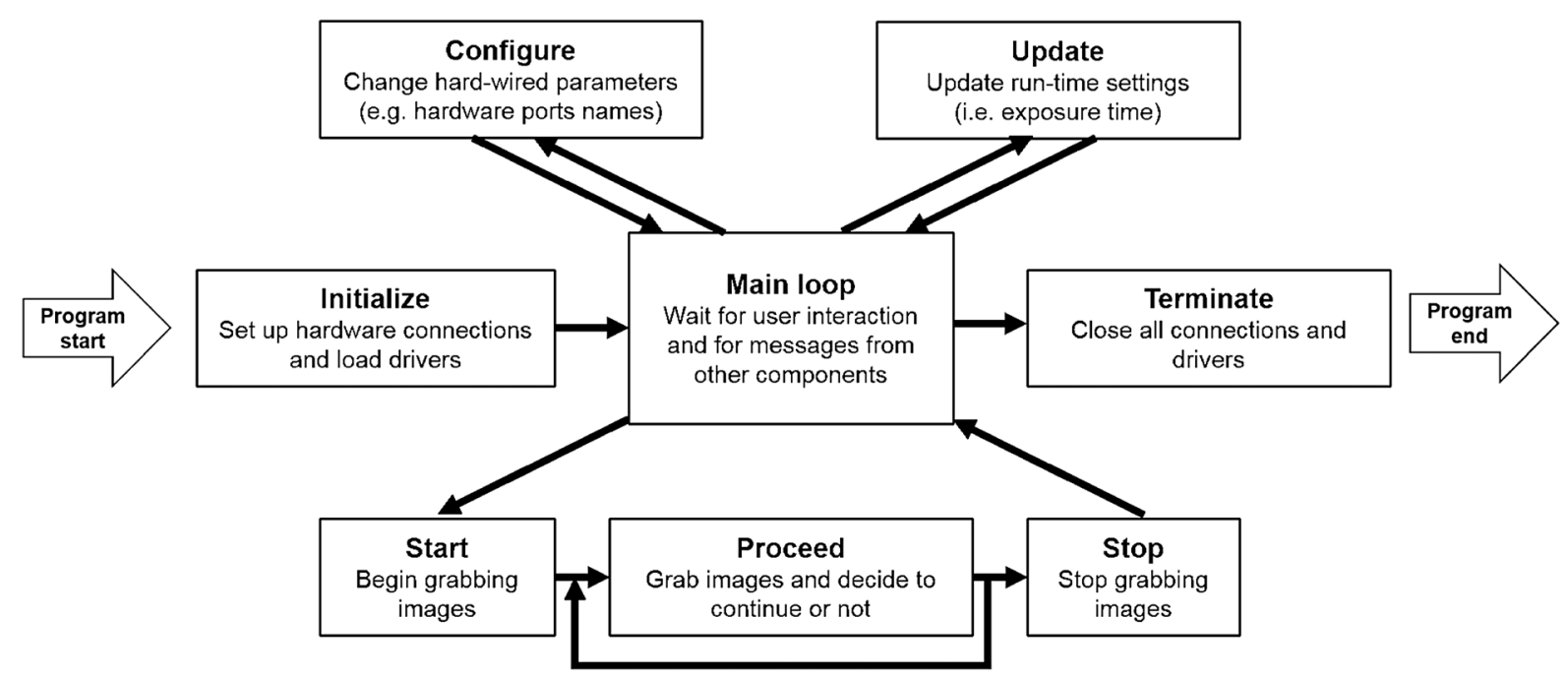

Fig. 9 Schematic view of the work flow of a representative Murmex component operating a camera. 
Each hardware component in the LSFM was managed through its own software component. Since the galvo scanners and the electro-optical modulator were not addressed directly but via analog and digital signals generated by the DAQ board, their software component just sent analog and digital waveforms to an additional intermediate component responsible for DAQ board management. Beyond hardware-related software, two additional software components, the Stack and Tomo components, orchestrated the operation of the entire system.

The Stack component was used to acquire image stacks along the detection axis, here the $z$ direction, and coordinates the Camera and the Stages components. Stacks are collected by continuously moving the sample along $z$ while keeping the camera acquiring in free run. The translation speed was calculated such that in the time needed to collect a single frame the specimen was moved by a $z$ step specified in the GUI. The Camera component used low-level libraries from Hamamatsu to save the data, producing one single. cxd file (the proprietary, noncompressed, tiff-like format from Hamamatsu) for each image stack. The Camera component must write data to disk with perfect synchronization, otherwise the nominal $z$ position of each frame is no more coincident with the real $z$ position of the plane imaged. For this reason, the process executing the Camera code was set to highest priority. Additionally, the Stack component checked the timing of data production, in order to re-acquire the image stack if the camera had encountered a timing issue.

The Tomo component is a high-level component which coordinated the acquisition of adjacent, parallel, partially overlapping stacks. The user can specify the volume to be imaged either by inserting the minimum and maximum $x, y$, and $z$ coordinates of a parallelepiped or by providing a. txt file with the coordinates of individual stacks to be collected. The Tomo component informs the experimenter via email when the acquisition is terminated either cleanly or through errors.

All the software described here can be freely downloaded from Ref. 35. Due to its modular structure and the extensive documentation, it can be easily integrated and adapted in other microscopy systems.

\section{Appendix E: Formulas}

In Table $2, f_{\mathrm{TL}}^{\text {design }}$ is the focal length of the tube lens for which the objective has been designed (200 mm for Leica and Nikon, $180 \mathrm{~mm}$ for Olympus and $165 \mathrm{~mm}$ for Zeiss), $f_{\mathrm{TL}}^{\text {real }}$ is the focal length of the tube lens actually employed, $M$ is the nominal magnification of the objective, $n$ is the refractive index of the immersion medium, $\lambda_{0}$ is the wavelength in vacuum, $\lambda$ is the wavelength in the medium, $w$ is the $1 / e^{2}$ radius of the Gaussian beam at the objective back focal plane, FN is the field number of the objective, $p$ is the physical pixel size, $r_{\text {chip }}$ is the size of the camera chip, $t_{\text {shutter }}$ is the pace of the rolling shutter (i.e., the time between exposure start in subsequent lines), and $t_{\exp }$ is the line exposure time. The subscripts $i$ and $d$ refer to illumination and detection, respectively.

\section{Acknowledgments}

The authors are grateful to Riccardo Ballerini and Ahmed Hajeb from the mechanics workshop at LENS for fabrication of the sample chamber. We further thank Marco De Pas from the electronic workshop for his expertize in the fabrication of the custom-made amplification electronics. We thank GARR for the 10-GB optical fibre connection to CINECA and CINECA for hosting our data. The research leading to these results has received funding from the European Union Seventh Framework Program (FP7/2007-2013) under grant agreements No. 604102 (Human Brain Project) and No. 284464 (LASERLAB-EUROPE). The research has also been supported by the Italian Ministry for Education, University, and Research in the framework of the Flagship Project NanoMAX, by "Ente Cassa di Risparmio di Firenze" (private foundation). Research activities were also supported by Regione Toscana in the program POR-CreO 2007-2013 (Linea di interventi 1.5.a-1.6Bando Unico R\&S 2012) under grant agreement (CUP) No. 6408.30122011.026000201. M.v. H has a financial interest in Murmex by Distrio, Amsterdam, the Netherlands.

\section{References}

1. J. Huisken et al., "Optical sectioning deep inside live embryos by selective plane illumination microscopy," Science 305(5686), 1007-1009 (2004).

2. P. Keller and H.-U. Dodt, "Light sheet microscopy of living or cleared specimens," Curr. Opin. Neurobiol. 22(1), 138-143 (2012).

3. H.-U. Dodt et al., "Ultramicroscopy: three-dimensional visualization of neuronal networks in the whole mouse brain," Nat. Methods 4(4), 331-336 (2007).

4. J. Huisken and D. Y. Stainier, "Selective plane illumination microscopy techniques in developmental biology," Development 136(12), 19631975 (2009)

5. J. Freeman et al., "Mapping brain activity at scale with cluster computing," Nat. Methods 11(9), 941-950 (2014).

6. R. Burns, J. Vogelstein, and A. Szalay, "From cosmos to connectomes: the evolution of data-intensive science," Neuron 83(6), 1249-1252 (2014).

7. P. Frasconi et al., "Large-scale automated identification of mouse brain cells in confocal light sheet microscopy images," Bioinformatics 30(17), i587-i593 (2014).

8. K. Chung et al., "Structural and molecular interrogation of intact biological systems," Nature 497(7449), 332-337 (2013).

9. R. Tomer et al., "Advanced CLARITY for rapid and high-resolution imaging of intact tissues," Nat. Protoc. 9(7), 1682-1697 (2014).

10. P. J. Keller and E. H. K. Stelzer, "Quantitative in vivo imaging of entire embryos with digital scanned laser light-sheet fluorescence microscopy," Curr. Opin. Neurobiol. 18(6), 624-632 (2008).

11. P. J. Keller et al., "Reconstruction of zebrafish early embryonic development by scanned light-sheet microscopy," Science 322(5904), 10651069 (2008).

12. E. Baumgart and U. Kubitscheck, "Scanned light-sheet microscopy with confocal slit detection," Opt. Express 20(19), 21805-21814 (2012).

13. I. Costantini et al., "A versatile clearing agent for multi-modal brain imaging," Sci. Rep. 5(9808), 1-9 (2015).

14. M. C. Teich and B. E. A. Saleh, Fundamentals of Photonics, Wiley Interscience, Canada (1991).

15. L. Silvestri et al., "Confocal light-sheet microscopy: micron-scale neuroanatomy of the entire mouse brain," Opt. Express 20(18), 20582-20598 (2012).

16. M. J. Booth and T. Wilson, "Refractive-index-mismatch induced aberrations in single-photon and two-photon microscopy and the use of aberration correction," J. Biomed. Opt. 6(3), 266-272 (2001).

17. T. Wilson and A. R. Carlini, "Size of the detector in confocal imaging systems," Opt. Lett. 12(4), 227-229 (1987).

18. G. Cox and C. J. Sheppard, "Practical limits of resolution in confocal and non-linear microscopy," Microsc. Res. Tech. 63(1), 1822 (2004).

19. M. Schwertner, M. J. Booth, and T. Wilson, "Characterizing specimen induced aberrations for high NA adaptive optical microscopy," Opt. Express 12(26), 6540-6552 (2004).

20. M. Schwertner, M. J. Booth, and T. Wilson, "Specimeninduced distortions in light microscopy," J. Microsc. 228(1), 97-102 (2007).

21. G. Castagna, Object-Oriented Programming a Unified Foundation, Birkhäuser, Boston (1997). 
22. N. Kovacevic et al., "A three-dimensional MRI atlas of the mouse brain with estimates of the average and variability," Cereb. Cortex 15(5), 639-645 (2004).

23. A. Bria and G. Iannello, "TeraStitcher-a tool for fast automatic 3Dstitching of teravoxel-sized microscopy images," BMC Bioinf. 13(1), 316 (2012).

24. Alessandro Bria, Leonardo Onofri, and Giulio Iannello, "TeraStitcher," http://abria.github.io/TeraStitcher/ (20 January 2015)

25. V. Marx, "Microscopy: seeing through tissue," Nat. Methods 11(12), 1209-1214 (2014).

26. K. Wang et al., "Rapid adaptive optical recovery of optimal resolution over large volumes," Nat. Methods 11(6), 625-628 (2014).

27. M. J. Booth, "Adaptive optics in microscopy," Trans. R. Soc. A 365(1861), 2829-2843 (2007).

28. R. Jorand et al., "Deep and clear optical imaging of thick inhomogeneous samples," PLoS One 7(4), e35795 (2012).

29. F. Fahrbach and A. Rohrbach, "A line scanned light-sheet microscope with phase shaped self-reconstructing beams," Opt. Express 18(23), 780-785 (2010)

30. T. Planchon et al., "Rapid three-dimensional isotropic imaging of living cells using Bessel beam plane illumination," Nat. Methods 8(5), 417423 (2011).

31. L. Gao et al., "3D live fluorescence imaging of cellular dynamics using Bessel beam plane illumination microscopy," Nat. Protoc. 9(5), 1083-1101 (2014).

32. "Murmex by distrio," in Software Framework for Distributed Input/ Output Applications, http://sine.ni.com/nips/cds/view/p/lang/it/nid/ 212895 (5 June 2015).

33. "LabbitMQ-Distrio," in Communicate with the RabbitMQ Message Broker, http://sine.ni.com/nips/cds/view/p/lang/it/nid/211065 (5 June 2015).

34. "RabbitM," in Robust Messaging for Applications, http://www.rabbitmq .com/RabbitMQ (5 June 2015).

35. Ludovico Silvestri, Caroline Müllenbroich, and Marcel van't Hoff, "Repository of Murmex components," https://github.com/marcel vanthoff/GiorgioGitHub (5 June 2015).

M. Caroline Müllenbroich graduated in physics at the University of Heidelberg, Germany, in 2008. She obtained her PhD at the Institute of Photonics, University of Strathclyde, Glasgow, Scotland, in 2012. She was a visiting scientist at Johns Hopkins Hospital, USA, where she worked on microscopy and imaging applications. She is currently a postdoc at the European Laboratory for Non-Linear Spectroscopy where she focuses on adaptive optics and light-sheet microscopy development.

Ludovico Silvestri received his master's degree in physics in 2008 at the University of Pisa. He then moved to the University of Florence, where he obtained a PhD in atomic and molecular spectroscopy in
2012. From 2012 to 2014, he has been a postdoc at the European Laboratory for Non-Linear Spectroscopy. Currently, he is a researcher at the National Institute of Optics in Florence. His research activity is focused on light-sheet microscopy and its applications.

Leonardo Onofri graduated in biomedical engineering at Università Campus Bio-Medico di Roma in 2010 and received his PhD in computer science from the same university in 2014. Currently, he is a postdoctoral researcher of medical imaging at the Katholieke Universiteit Leuven. His research interests include biomedical and biological data, signal and image processing, computer vision, pattern recognition, and medical imaging.

Irene Costantini obtained her master's degree in medical and pharmaceutical biotechnology in 2011 at the University of Florence with professor Cavalieri and professor Netea. The internship was conducted in the Radboud University Nijmegen Medical Center, Netherlands. She is currently attending an international PhD in atomic and molecular photonics at LENS. Her research activity is focused on 3-D reconstruction of intact neuronal networks in whole mouse and human brains using light-sheet microscopy and two-photon fluorescence microscopy.

Marcel van't Hoff: Biography is not available.

Leonardo Sacconi received his master's degree in physics in 2001 at the University of Florence. He then moved to Trento where he obtained a PhD in physics. He was a visiting scientist at the Cornell University, Ithaca, New York, USA, in the group of Watt Webb. From 2005 to 2011, he started new research lines on functional imaging of neuronal networks in vivo. He is now a researcher at the National Institute of Optics in Florence.

Giulio lannello graduated in electronic engineering at Politecnico di Milano in 1981 and received his PhD in computer science and computer engineering from the University of Napoli Federico II in 1987. Currently, he is a full professor of computer science and computer engineering at the University Campus Bio-Medico di Roma. His current research interests include biomedical and biological data, signal and image processing, bioinformatics, and performance evaluation of parallel and distributed algorithms and systems.

Francesco S. Pavone is developing new microscopy techniques for high-resolution and high-sensitivity imaging and for laser manipulation purposes. These techniques have been applied to single-molecule biophysics, single-cell imaging and optical manipulation. Tissue imaging is another research area developed, where nonlinear optical techniques have been applied for skin and neural tissue imaging also in vivo. He is currently director of the European Laboratory for Non-Linear Spectroscopy in Florence. 